\section{A dairy worker with fever and an abnormal echocardiogram}

\section{DESCRIPTION OF THE CASE}

A 38-year-old male presented with history of progressively increasing dyspnoea of 25 days duration. He gave history of low -grade fever associated with malaise and weight loss over the preceding 6 months. He worked in the dairy industry in the Middle East and returned to India owing to his illness. On clinical examination, he was found to be tachypneic and cachectic. Jugular venous pressure was raised with a prominent 'a' wave. There was a short early diastolic murmur over the aortic area. His blood investigations, including renal and liver function tests, were normal. Three sets of blood cultures were sterile. Two-dimensional trans-thoracic and trans-oesophageal echocardiography revealed thickened bicuspid aortic valve cusps, with moderate eccentric aortic regurgitation and an abnormal structure posterior to the left ventricular outflow tract and aorta (figure $1 \mathrm{~A}-\mathrm{C}$ ). A small vegetation was seen attached to the fused right-left aortic cusp (supplementary figure 1). The patient was started on appropriate intravenous antibiotics and antifailure medications, and was referred for early surgical treatment.

\section{QUESTION}

Identify the structure depicted in the images (figure 1A-C).

Answer options:

A. Cor triatriatum

B. Aortic dissection

C. Left atrial pseudoaneurysm

D. Pseudoaneurysm of the mitral aortic inter-valvular fibrosa

E. Aortic root abscess.
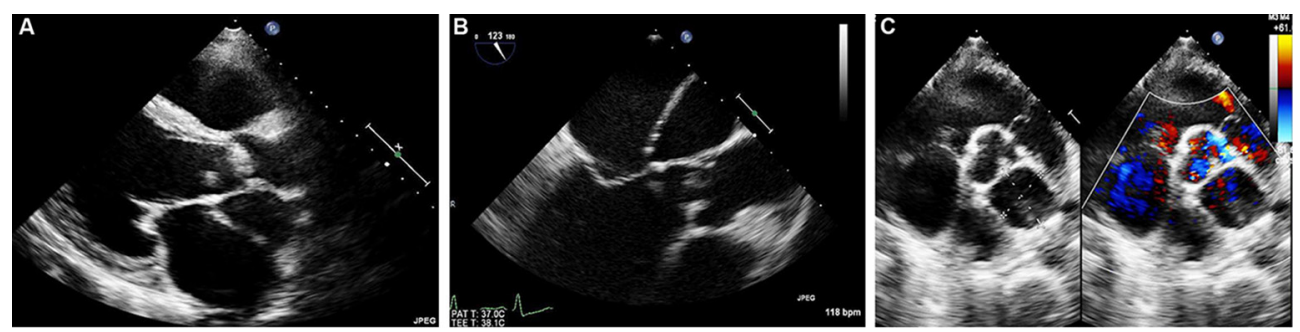

Figure 1 (A) Transthoracic echocardiography parasternal long axis view. (B) Transesophageal echocardiography (mid esophageal level) long axis view. (C) Transthoracic echocardiography parasternal short axis view. 


\section{Answer: Option d}

A large pseudoaneurysm (PsA) is seen arising from mitralaortic intervalvular fibrosa (MAIVF) region (figure 1A, B; see online supplementary video 1 ). PsA is situated posterior to the aorta (figure 1C) and is seen communicating with the left ventricular outflow tract (see online supplementary video 2, supplementary video 3), adjacent to the left coronary cusp (supplementary video 3). There was a vegetation at the site of the breach in the MAIVF. Cardiac MRI (see online supplementary figure 2, supplementary figure 3 ) revealed that the pseudoaneurysm (PsA-MAIVF) measured $6.4 \mathrm{~cm} \times 4 \mathrm{~cm} \times 3.6 \mathrm{~cm}$ and was sandwiched between the aorta anteriorly and the left atrium posteriorly. CT angiogram (see online supplementary figure 4, supplementary figure 5) showed superiorly the PsA extended above the level of left pulmonary artery and inferiorly it was in close proximity to the left atrioventricular groove.

Absence of an intimal breach in the aortic root and visualisation of a clear communication tract above the level of aortic cusps rule out aortic dissection.

Aortic root abscesses are usually located anterior to the aorta, although in rare instances they can be posterior. The abscess cavity communicates with the aortic root with the communication tract usually located above the level of the valve cusps. ${ }^{1}$ An aortic root abscess in comparison to PsA is small and non-pulsatile in nature.

Left atrial pseudoaneurysm commonly originates from the atrial appendage and is commonly associated with infective endocarditis involving the mitral valve or following blunt trauma.

Cor triatriatum is seen as a fibromuscular membrane, partitioning the left atrial cavity. The two chambers communicate with a variable size orifice with evidence of diastolic doming with flow of blood. The absence of the above finding and the presence of a clear communication with the left ventricular outflow, with filling of the chamber in systole, exclude this diagnosis.

The MAIVF tissue, being relatively avascular, is commonly involved in endocarditis and can result in pseudoaneurysm formation. ${ }^{2}$ The causative organism was identified to be Brucella species based on elevated serum agglutination test antibody titres. The patient was started on treatment with doxycycline and streptomycin and stabilised with antifailure medications.
He was advised to undergo early surgery after completion of 2 weeks of antibiotic therapy.

Brucellosis is prevalent in the Middle-Eastern region among people who are in close contact with cattle and dairy products, and is an important cause of culture negative infective endocarditis with preferential aortic valve involvement. ${ }^{3}$ This case highlights a rare cause of pseudoaneurysm of the MAIVF complicating Brucella endocarditis.

\section{Gopal Chandra Ghosh, Lijo Varghese, Viji Samuel Thomson}

Department of Cardiology, Christian Medical College and Hospital, Vellore, India

Correspondence to Dr Gopal Chandra Ghosh, Department of Cardiology, Room No. 310, Hospital Annexe, Christian Medical College and Hospital campus, Vellore, India; gcghosh86@gmail.com

Contributors All the authors were involved in the clinical management of the patients. GCG and LV drafted, and VST revised the manuscript.

Competing interests None declared.

Patient consent Obtained.

Ethics approval Institutional Review Board.

Provenance and peer review Not commissioned; internally peer reviewed.

(c) Article author(s) (or their employer(s) unless otherwise stated in the text of the article) 2017. All rights reserved. No commercial use is permitted unless otherwise expressly granted.

- Additional material is published online only. To view please visit the journal online (http://dx.doi.org/10.1136/heartasia-2017-010915).

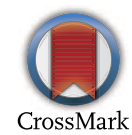

To cite Ghosh GC, Varghese L, Thomson VS. Heart Asia 2017;9:1-2.

Heart Asia 2017:9:1-2. doi:10.1136/heartasia-2017-010915

\section{REFERENCES}

1 Brecker SJ, Pepper JR, Eykyn SJ. Aortic root abscess. Heart 1999;82:260-2.

2 Sudhakar S, Sewani A, Agrawal M, et al. Pseudoaneurysm of the mitral-aortic intervalvular fibrosa (MAIVF): a comprehensive review. J Am Soc Echocardiogr 2010:23:1009-18

3 Lulu AR, Araj GF, Khateeb MI, et al. Human brucellosis in Kuwait: a prospective study of 400 cases. Q J Med 1988:66:39-54. 